Hydrol. Earth Syst. Sci., 12, 1121-1127, 2008

www.hydrol-earth-syst-sci.net/12/1121/2008/

(C) Author(s) 2008. This work is distributed under

the Creative Commons Attribution 3.0 License.

\title{
Estimation of streamflow by slope regional dependency function
}

\author{
A. Altunkaynak \\ Istanbul Technical University, Faculty of Civil Engineering, Maslak 34469, Istanbul, Turkey \\ Received: 20 February 2008 - Published in Hydrol. Earth Syst. Sci. Discuss.: 11 April 2008 \\ Revised: 18 June 2008 - Accepted: 9 July 2008 - Published: 25 August 2008
}

\begin{abstract}
Kriging is one of the most developed methodologies in the regional variable modeling. However, one of its drawbacks is that the influence radius can not be determined by this method. In which distance and in what ratio that pivot station is influenced from adjacent sites is rather often encountered problem in practical applications. Regional weighting functions obtained from available data consist of several broken lines. Each line has different slopes which represent the similarity and the contribution of adjacent stations as a weighting coefficient. The approach in this study is called as Slope Regional Dependency Function (SRDF). The main idea of this approach is to express the variability in value differences $\gamma$ and distances together. Originally proposed SRDF and Trigonometric Point Cumulative SemiVariogram (TPCSV) methods are used to predict streamflow. TPCSV and Point Cumulative Semi-Variogram (PCSV) approaches are also compared with each other. Prediction performance of all the three methods revealed a relative error less than $10 \%$ which is acceptable for most engineering applications. It is shown that SRDF outperforms PCSV and TPCSV with very high differences. It can be used for missing data completion, determination of measurement sites location, calculation of influence radius, and determination of regional variable potential. The proposed method is applied for the 38 stream flow measurement sites located in the Mississippi River basin.
\end{abstract}

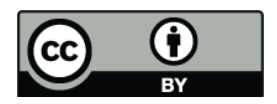

Correspondence to: A. Altunkaynak (altunkay@itu.edu.tr)

\section{Introduction}

The quantity of streamflow plays a significant role on planning, management and design of the water resources. Discharge is directly related with reservoir operation, forecasting of floods and droughts, hydroelectric power production, irrigation, protection of ecosystem and sedimentation. Therefore, prediction and calculation of the discharge are very important.

Kriging is the most developed regional prediction method. It is originally proposed by Krige (1951), who is a South African engineer, established the principles of spatial prediction theory. Matheron $(1963,1971)$ contributed to development of regional variable theory. Although Kriging is one of the most developed regional prediction method, it fails to define the influence radius which delineate the borders of the contribution areas. Kriging method has been applied to mining (Matius et al., 2004), tunnel (Öztürk and Nasuf, 2002), hydrology (Altunkaynak et al., 2003; Şen and Habib, 1998), hydraulics (Altunkaynak et al., 2005) and ocean engineering (Altunkaynak, 2005; Altunkaynak and Özger, 2005). This approach is also often used in geostatistics to determine the parameters of regional variability. The principles of geostatistics are based on the theory that sites close to each other exhibit similar features but the correlation decreases when the distance between sites increase. The semivariogram which is an important parameter in geostatistics shows the correlation between measurement sites. The semivariogram proposed by Matheron (1963) includes assumptions that are stationary and measurement points should be in equal distances. This can not be used until the assumptions are fulfilled. However, in nature the measurement sites are scattered irregularly in the region rather than located at regular grid points. Huang and Yang (1998) used Kriging methodology to estimate discharge assumed as regional variable. However, it is not possible to determine influence radius by this method.

Published by Copernicus Publications on behalf of the European Geosciences Union. 


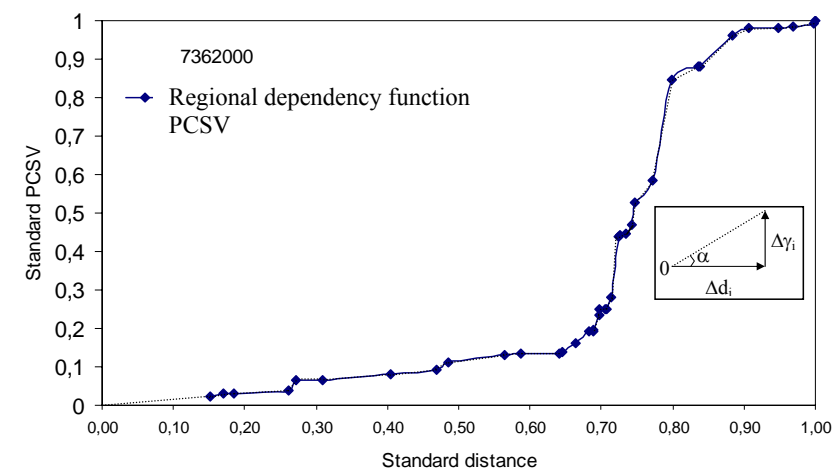

Fig. 1. Regional Dependency Function according to PCSV.

Peng and Buras (2000) used spatial method to estimate discharge for multi-reservoir operation. In case of non-stationarity and irregularity of measurement points, Şen (1989) proposed Point Cumulative Semi Variogram (PCSV) approach based on relationship between point and area. It is possible to determine influence radius from selected point by standard regional dependence procedure introduced by Şen and Habib (1998). To determine the effective range and weighting coefficient of a variable is very important for estimations and calculations. PCSV method is based on the differences between selected pivot point and the surrounding points. The recorded variables in the points constitute differences that lead to regional dependence functions. These functions can be used for missing data completion, optimum location of measurement sites, calculation of influence radius and determination of regional variable potential. Şahin and Şen (2004) developed Trigonometric Point Cumulative Semi Variogram (TPCSV) technique and applied to wind data. Also, Altunkaynak (2005) applied this method for the estimation of ocean wave characteristics.

The aim of the study is to estimate the station values from neighboring stations by using Slope Regional Dependency Function (SRDF). The proposed method is applied for the stream flow measurement network located in the Mississippi River basin.

\section{Regional Dependency Function (RDF)}

PCSV method is based on the half square differences $[\gamma(d)]$ between pivot site and randomly scattered adjacent sites. This approach searches the effect of one point to the other points. It is possible to obtain data of stations which are not measured or missing by using regional weighting functions. Moreover, influence radius of each site can be determined by these functions. If the difference is very high between two stations then the next station would not be taken into consideration in calculations. The relationship between points can

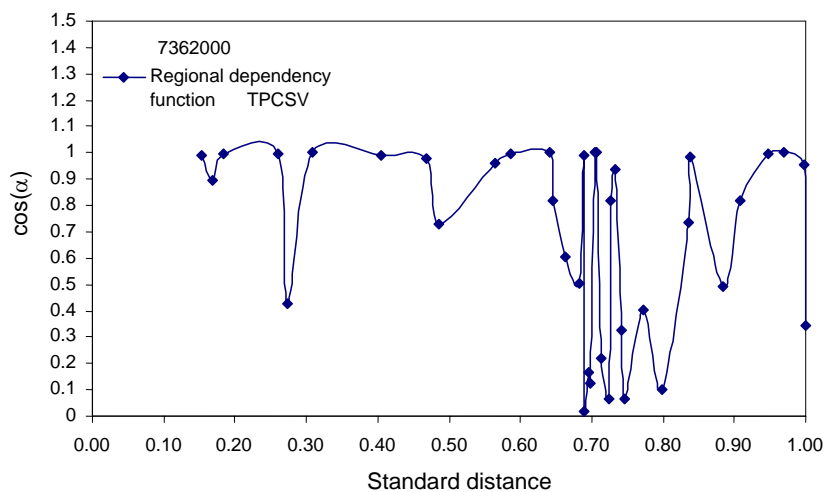

Fig. 2. Regional Dependency Function according to TPCSV.

be given as following equation.

$\gamma\left(d_{i}\right)=\frac{1}{2}\left(q_{p}-q_{d i}\right)^{2}(i=1,2, \cdots n)$

Where $q_{p}$ and $q_{d}$ are pilot station and adjacent stations discharge values, respectively. $n$ is the number of stations. When it is desired to show the variation of PCSV values by distance on a Cartesian coordinate system, the $\mathrm{x}$-axis represents the distances from pivot point and the y-axis represents the PCSV values. For the sake of simplicity, Eq. (1) should be standardized. After this operation, $x$ - and y-axes take the values ranging from 0 to 1 . Standardization can be achieved by dividing the all values to the maximum. It can be said that as the distance increases the correlation between pivot and other points decreases and also the weighting coefficient which represents the contribution of adjacent points decreases. The weightings of the highly correlated points approach to 1 . On the other hand it takes 0 for uncorrelated points.

Şahin ve Şen (2004) applied TPCSV technique to the wind data. This approach was also developed by considering correlation principle. Two different data array can be put in the same set when they are highly correlated. Two data array, $\boldsymbol{x}=\left(a_{11}, a_{12}, a_{13}, \cdots a_{1 n}\right)$ and $\boldsymbol{y}=\left(a_{21}, a_{22}, a_{23}, \cdots a_{2 n}\right)$ are the vectors which have the same initial point. The angle between two vectors is required for determination of similarity of two data arrays or vectors. Scalar multiplication of $\boldsymbol{x}$ and $\boldsymbol{y}$ vectors is indicated as below.

$\cos \alpha=\frac{\vec{x} \cdot \vec{y}}{|\vec{x}| \cdot|\vec{y}|}$

The nominator of this expression corresponds to sum of multiplication of vector reciprocal components. The denominator is equal to multiplication of two vector norms. So, if each vector is divided by its norms, then unit vectors would be obtained. In fact, $\cos \alpha$ is equal to summation of unit vector multiplications that correspond to each other. $\cos \alpha$ shows the similarity between two points or two arrays. In other words, it can be defined as correlation coefficient. 
As the angle is narrower, these two points or arrays show more similarity. If the angle is zero, it can be said that there is an exact dependency between these points or arrays and the correlation coefficient is equal to 1 . In contrast, if the angle is $90^{\circ}$ two points or arrays have no dependency and the correlation coefficient is equal zero. Consequently as the angle increases, the similarity reduces. The regional dependence function depicted in Fig. 1 shows $\cos \alpha$ values of stations found in the $d$ distance from pivot point and contributions of these points to pivot point. The point selected in the figure is shown as,

$\Delta d_{i}=d_{i+1}-d_{i}$,

where $\Delta d$ is the distance difference between two consecutive points. The $\gamma(d)$ between two consecutive points on y-axis of the same figure is shown as $\Delta \gamma$.

$\Delta \gamma_{i}=\gamma\left(d_{i+1}\right)-\gamma\left(d_{i}\right)$

Hypotenuse value is required to determine the value of $\cos \alpha$. The square root of summation squares of Eqs. (3) and (4) give hypotenuse.

$$
|A B|_{i}=\sqrt{\left(\Delta d_{i}\right)^{2}+\left(\Delta \gamma_{i}\right)^{2}}
$$

$|A B|$ is the length of the line which is constituted by two points. All $\cos \alpha$ values in the same figure can be calculated as following.

$\left(\cos \alpha_{i}\right)_{i}=\frac{\Delta d_{i}}{|A B|_{i}}$

The number of $\cos \alpha$ value calculated is $n-1$ which corresponds to $n$ points. The angle range is from $0^{\circ}$ to $90^{\circ}$, because regional dependency function increases monotony. The weighted average is used in the prediction of pivot station discharge value. The summation of $\cos \alpha$ values is,

$L=\cos \alpha_{1}+\cos \alpha_{2}+\cdots \cos \alpha_{n}$.

Where $L$ is sum of the $\cos \alpha$ values. Following expression is given for the prediction of pivot point value.

$\overline{q_{p}}=\frac{1}{L} \sum_{i=1}^{N} q_{i} \cdot \cos \alpha_{i}$

Here $\cos \alpha$ is expressed as the weighting coefficient. The regional dependency function shown in Fig. 1 increases continuously by the distance. Function curve exhibits distinct features in different portions of distances such as it rise more linearly, increasingly or decreasingly. Regional dependency function consists of several lines. Slope of the each line $(\tan \alpha)$ explains the similarity between two points. It also gives information about the regional dependency. The ratio of the $\gamma(d)$ differences, $\Delta \gamma(d)$ to distance differences leads to slope which is the first derivation of line. If the differences between the points are small then this means that the slope will reduce and dependency will increase. On contrary, steep slope means weak dependency. Both $\gamma(d)$ and distance variations are taken into account together by considering the slope regional dependency function (SRDF) which is the main idea of this study. Closeness to pivot point does not mean that they are very similar to each other and their correlation is high. The square of differences $\left(q_{p}-q_{d}\right)^{2}$ between pivot point and close points should be low. Here $q_{p}$ and $q_{d}$ are the runoff depths at pivot point and the point at $d$ distance, respectively. When distance and $\gamma(d)$ variations are low, it can be said that these two points are very similar and their contribution to value of pivot point prediction are very high. The effect of both $\gamma(d)$ differences, $\Delta \gamma(d)$ and the distance differences between two points, $\Delta d$, is considered together in this approach. The ratio of $\Delta \gamma(d)$ to $\Delta d$ gives dimensionless slope.

Dependency factor $=\tan \alpha=\frac{\Delta \gamma(d)}{\Delta d}$

when $\tan \alpha$ value between two points is lower, it contributes more to the weighting average of pivot point. Therefore, regional dependency function slope identified as dependency factor of this point. The slope $(\tan \alpha)$ of regional dependency function gives the similarity and dependency. The flatter slope contributes to pivot point with high weighting coefficient. In contrast, the contribution of point to the pivot point is very low when the slope is very steep. There is an inverse relationship between slope and adjacent sites contribution. The weighting coefficient that represents the contribution of a point is equal to $\cot \alpha$.

Weighting coefficient $=\cot \alpha_{i}=\frac{\Delta d}{\Delta \gamma(d)}$

$\cot \alpha$ is used instead of $\cos \alpha$ which is employed for TPCSV method for prediction of the pivot point value. The summation of $\cot \alpha$ values used in weighted average is given as below.

$m=\cot \alpha_{1}+\cot \alpha_{2}+\cdots \cot \alpha_{n}$

Here $m$ is the summation of weighting coefficients. $\cos \alpha$ in Eq. (8) used for TPCSV method is replaced by $\cot \alpha$ to predict the pivot point discharge value.

$\overline{q_{p}}=\frac{1}{m} \sum_{i=1}^{N_{d}} q_{i} \cdot \cot \alpha_{i}$

Here, $\overline{q_{p}}$ is runoff depth value of the predicted pivot point. Altunkaynak et al. (2005) reported the same prediction results by using PCSV method. Our study is the first in which TPCSV and SRDF methods are applied for the 38 stations located in Mississippi River basin. In this study, the purpose is to interpret the consequences obtained from an application of a new (presented) method and to compare these results with other methods in the literature. 
Table 1. Station 7362000 Detailed Prediction Calculations According to SRDF.

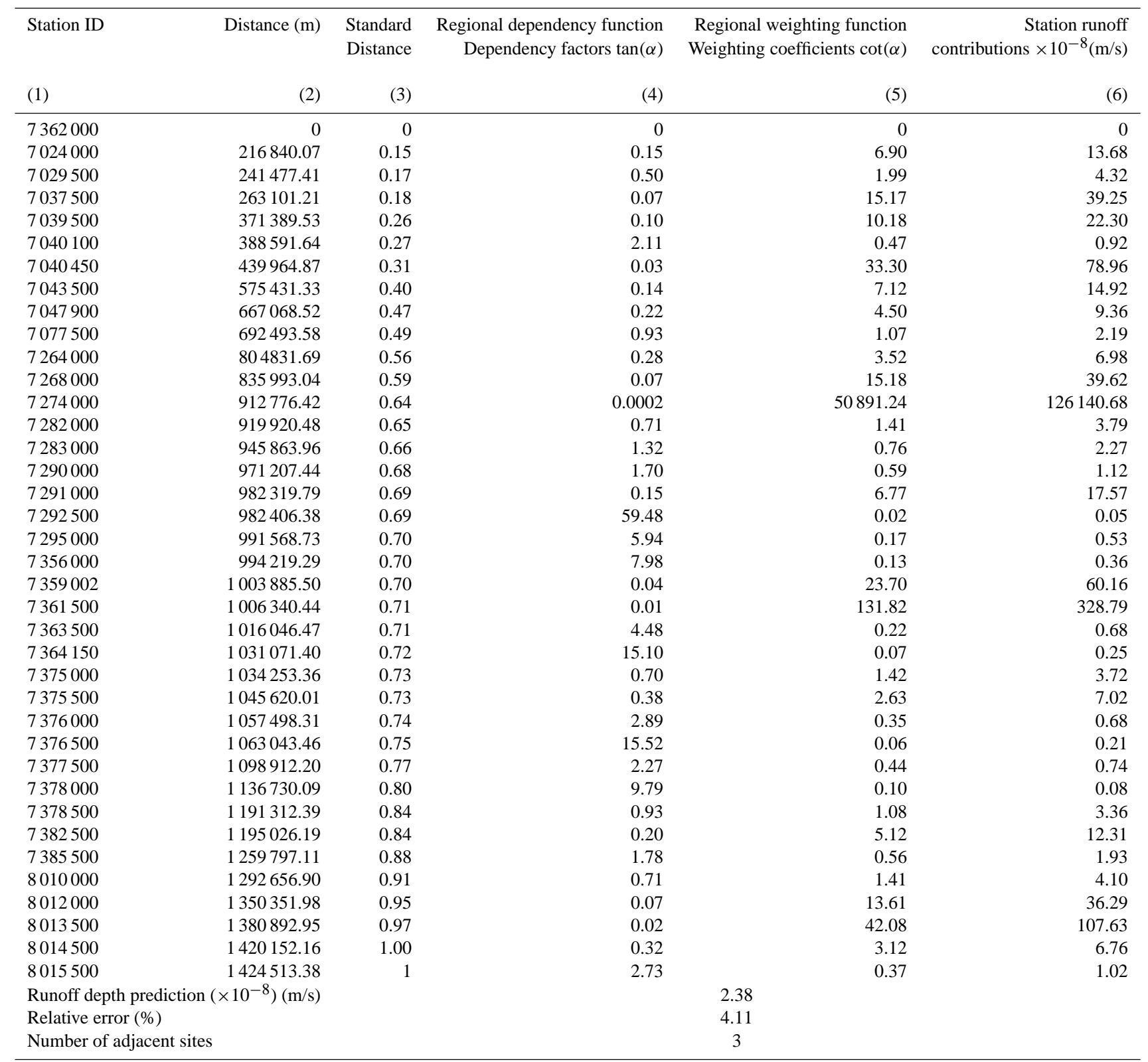

\section{Application}

In this study, 38 measurement stations located in Mississippi River basin are used. Data is obtained from the study of Altunkaynak et al. (2005). The aim of using the same data is to compare PCSV, TPCSV and SRDF methods under equal conditions. Altunkaynak et al. (2005) computed runoff depth values of 38 measurement stations by using PCSV technique. Here, TPCSV and proposed model are employed for the same data. Study area, location map and detailed information on PCSV technique can be found in the study of Altunkaynak et al. (2005). In SRDF method, and for the pivot station 7362000 distances from pivot station, standard distances, $\tan \alpha=$ dependency factor and $\cot \alpha=$ weighting coefficient are given in Table 1 at second, third, fourth and fifth columns, respectively. Contributions of surrounding stations to the pivot station 73620000 , predicted runoff depth, relative errors and number of adjacent sites are included in Table 1. Regional dependency function of station 7362000 is shown in Fig. 1. This function increase continuously by standard distance as shown in Fig. 1. The curve in the figure consists of three portions. In the first part, it ranges from 0 to 0.7 standard distances and there is a linear increasing trend. 


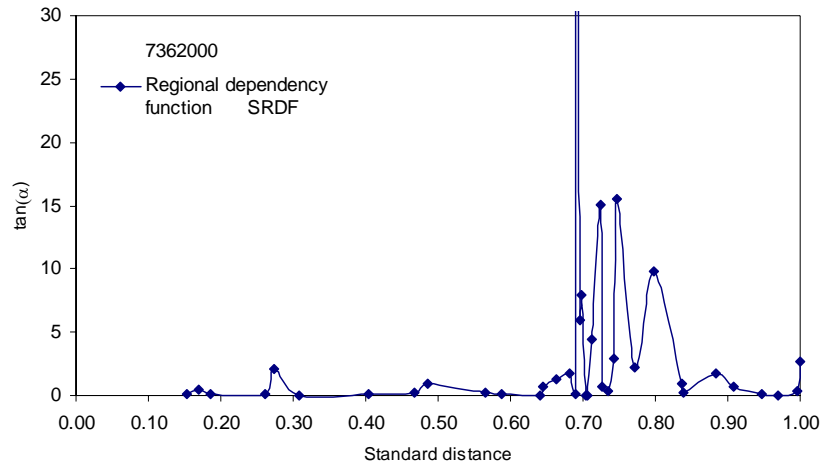

Fig. 3. Regional Dependency Function according to SRDF.

In the second part, it is between 0.7 and 0.8 , and increment is seen. Finally in the last part there is a decreasing trend. There is linear trend in the first part, because the slope approaches to a constant value or it fluctuates around a constant value. When one looks at the part of the dependency function which corresponds to $0-0.7$ standard distance at horizontal axis in Fig. 3, low fluctuations of $\tan \alpha=$ dependency function around a mean value can be seen. On the other hand, there is an agreement with the weighting coefficient which corresponds to same range in Fig. 4. Also, it can be said that this region exhibits a homogeny structure. The expression of homogeny structure means that stations found in this region are similar to each other and have strong regional dependency. The increasing rise of regional weighting function in Fig. 1 corresponds to a portion $(0.7-0.8)$ which has big fluctuations in Fig. 3. There is heterogenic structure in this part. The stations found in this region have no similarities and dependencies with pivot station. Although there is a decreasing rise trend in the third portion of Fig. 1, deviations from the mean are less than second portion shown in Figs. 3 and 4. The similarities and correlations to pivot station in this part are more than the second part. Mississippi River Basin can be divided into three part based on regional weighting function. Since the second part has high heterogeneity, it can be divided into more sub regions. This can be an input for the integrated basin management. Figs. 1, 3 and 4 demonstrate that which distances are homogeny or heterogenic in the first, second and third parts. The influence radius can be determined by this way. All these interpretations can be made by using Fig. 2 that shows the dependency function. Also, three parts are seen obviously from this figure too. Low deviations from the mean value at $0-0.7$ standard distance, high variability of dependency at $0.7-0.8$ and lower variation of dependency comparing to second part at $0.8-1.0$ are also valid in this figure. It is possible to predict missing or unmeasured runoff depths by using these functions. Let it is assumed that runoff depth data for the station 7362000 is unavailable or missing.

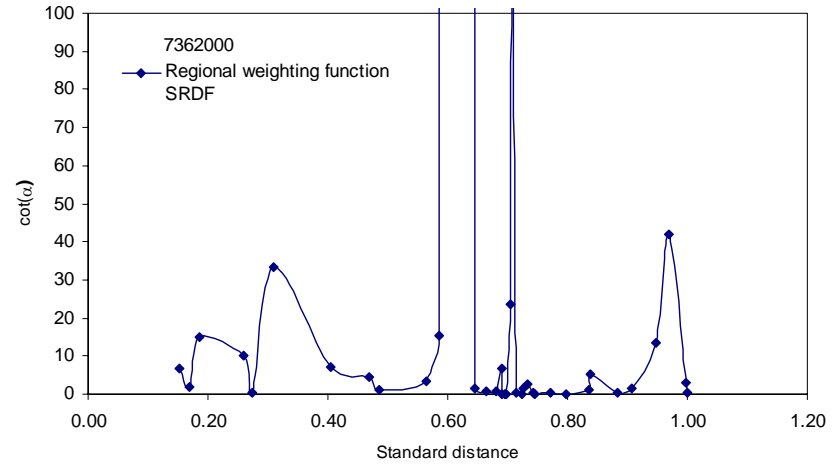

Fig. 4. Regional Weighting Function according to SRDF.

Firstly, distances from pivot station to others are calculated and standardized by dividing all one to maximum distance. Distance between the stations 7362000 and 8015500 is the maximum, and it is indicated in the second column of the Table 1. In the fourth column of the Table 1, regional weighting function calculated from Eq. (9) is available. In the fifth column, there is a weighting coefficient obtained from Eq. (10) and the last column indicates the contributions of adjacent stations to the pivot station. Fig. 4 shows the weighting coefficients for the station 7362000 . It can be seen from the figure that high contributions are expected from the stations in $0-0.7$ standard distance range. For the range $0.7-0.8$, the weighting factor is too small and for $0.8-1$, it is relatively bigger than the former range. The value of the pivot station 7362000 which has the relative error of $4.11 \%$ is the best predicted from three closest adjacent sites. The predicted value for this station is $2.38 \mathrm{~m} / \mathrm{s}$. The influence radius $R$ is equal to $263101.21 \mathrm{~m}$ that corresponds to distance of the third station to pivot station. Table 2 presents the predictions, relative errors and number of adjacent sites that give the best results obtained from PCSV, TPCSV and SRDF techniques. In Table 2, second column shows observations; third column shows the predicted values by PCSV method and fourth column reveals the predicted values by SRDF which is proposed in this study. Sixth, seventh and eighth columns are the prediction errors. Finally, ninth, tenth and eleventh columns present the number of adjacent sites which give the best results. Star symbol at eighth column indicates that SRDF predicts better than the PCSV. When this column is considered, it is shown that SRDF outperforms PCSV at 28 out of 38 stations. The mean relative errors are $3.07 \%$ and $7.79 \%$ in SRDF and PCSV, respectively. The ratio of better performance is at the level of $61 \%$. Similarly, it can be seen in the sixth column of Table 2 that 10 out of 38 stations prediction errors are greater than $10 \%$ by PCSV method. On the other hand, SRDF method has prediction error that is greater than $10 \%$ at 5 out of 38 stations. This corresponds to $50 \%$ better performance. When the aforementioned criteria are repeated again, it is seen that SRDF also outperforms TPCSV. 
Table 2. Comparison of PCVS, TPCVS and SRDF methods.

\begin{tabular}{|c|c|c|c|c|c|c|c|c|c|c|}
\hline \multirow{2}{*}{$\begin{array}{l}\text { Station ID } \\
\text { (1) }\end{array}$} & \multirow{2}{*}{$\begin{array}{l}\text { Runoff depth } \\
10^{-8}(\mathrm{~m} / \mathrm{s}) \\
\text { (2) }\end{array}$} & \multicolumn{3}{|c|}{$\begin{array}{l}\text { January runoff depth prediction } 10^{-8} \\
(\mathrm{~m} / \mathrm{s})\end{array}$} & \multicolumn{3}{|c|}{ Relative error (\%) } & \multicolumn{3}{|c|}{ Number of adjacent site } \\
\hline & & $\begin{array}{l}\text { PCSV } \\
\text { (3) }\end{array}$ & $\begin{array}{l}\text { TPCSV } \\
\text { (4) }\end{array}$ & $\begin{array}{l}\text { SRDF } \\
(5)\end{array}$ & $\begin{array}{r}\text { PCSV } \\
(6)\end{array}$ & $\begin{array}{r}\text { TPCSV } \\
(7)\end{array}$ & $\begin{array}{r}\text { SRDF } \\
(8)\end{array}$ & $\begin{array}{r}\text { PCSV } \\
(9)\end{array}$ & $\begin{array}{r}\text { TPCSV } \\
(10)\end{array}$ & $\begin{array}{r}\text { SRDF } \\
(11)\end{array}$ \\
\hline 7024000 & 2.41 & 2.26 & 2.27 & 2.37 & 6.33 & 8.95 & $1.36^{*}$ & 4 & 9 & 9 \\
\hline 7029500 & 3.31 & 2.81 & 2.48 & 3.13 & 15.18 & 24.75 & $5.33 *$ & 2 & 9 & 2 \\
\hline 7037500 & 1.68 & 1.95 & 1.92 & 1.91 & 14.04 & 13.02 & $12.47 *$ & 3 & 2 & 2 \\
\hline 7039500 & 1.96 & 1.99 & 1.98 & 1.97 & 1.65 & 1.20 & $0.81 *$ & 4 & 4 & 3 \\
\hline 7040100 & 1.90 & 1.87 & 1.87 & 1.95 & 1.72 & 1.30 & 2.75 & 3 & 3 & 3 \\
\hline 7040450 & 1.98 & 2.01 & 2.00 & 1.97 & 1.63 & 1.39 & $0.46^{*}$ & 3 & 3 & 5 \\
\hline 7043500 & 2.05 & 2.05 & 2.05 & 2.04 & 0.01 & 0.14 & 0.38 & 5 & 3 & 3 \\
\hline 7047900 & 2.08 & 2.04 & 2.08 & 2.09 & 1.58 & 0.09 & $0.54 *$ & 5 & 4 & 3 \\
\hline 7077500 & 2.09 & 2.11 & 2.10 & 2.08 & 0.52 & 0.61 & 0.67 & 3 & 3 & 2 \\
\hline 7264000 & 2.19 & 2.19 & 2.18 & 2.14 & 0.01 & 0.55 & 2.05 & 4 & 4 & 7 \\
\hline 7268000 & 3.14 & 2.73 & 2.73 & 3.08 & 12.89 & 12.81 & $1.72 *$ & 4 & 4 & 4 \\
\hline 7274000 & 2.48 & 2.49 & 2.61 & 2.53 & 0.44 & 5.12 & 2.23 & 9 & 6 & 5 \\
\hline 7282000 & 2.54 & 2.54 & 2.54 & 2.53 & 0.01 & 0.06 & 0.02 & 8 & 2 & 9 \\
\hline 7283000 & 2.60 & 2.59 & 2.60 & 2.60 & 0.01 & 0.16 & 0.46 & 7 & 8 & 5 \\
\hline 7290000 & 2.61 & 2.60 & 2.66 & 2.61 & 0.42 & 2.06 & $0.21 *$ & 7 & 3 & 2 \\
\hline 7291000 & 2.62 & 2.78 & 2.69 & 2.61 & 5.91 & 2.82 & $0.23 *$ & 4 & 2 & 4 \\
\hline 7292500 & 2.49 & 2.76 & 2.74 & 2.64 & 9.52 & 9.21 & $5.67 *$ & 2 & 2 & 2 \\
\hline 7295000 & 2.89 & 2.88 & 2.87 & 2.91 & 0.38 & 0.55 & 0.68 & 6 & 6 & 3 \\
\hline 7356000 & 2.37 & 2.42 & 2.38 & 2.37 & 1.81 & 0.20 & $0.04 *$ & 3 & 2 & 7 \\
\hline 7359002 & 2.59 & 2.31 & 2.32 & 2.34 & 10.97 & 10.38 & $9.38^{*}$ & 4 & 4 & 4 \\
\hline 7361500 & 2.17 & 2.33 & 2.26 & 2.19 & 7.04 & 3.98 & $1.12 *$ & 5 & 6 & 4 \\
\hline 7362000 & 2.48 & 2.24 & 2.25 & 2.38 & 9.69 & 9.33 & $4.11^{*}$ & 3 & 3 & 3 \\
\hline 7363500 & 1.98 & 2.20 & 2.20 & 1.95 & 9.95 & 9.90 & $1.61 *$ & 8 & 3 & 3 \\
\hline 7364150 & 1.95 & 2.14 & 2.14 & 1.98 & 9.18 & 9.36 & $2.01 *$ & 6 & 6 & 2 \\
\hline 7375000 & 2.17 & 2.68 & 2.70 & 2.60 & 19.18 & 19.75 & $17.33^{*}$ & 3 & 9 & 2 \\
\hline 7375500 & 2.79 & 3.17 & 2.78 & 2.76 & 12.07 & 0.27 & $0.91 *$ & 2 & 6 & 7 \\
\hline 7376000 & 2.67 & 2.76 & 2.73 & 2.64 & 3.17 & 2.49 & $0.8^{*}$ & 3 & 2 & 4 \\
\hline 7376500 & 2.56 & 2.54 & 2.57 & 2.65 & 1.28 & 0.68 & 3.62 & 3 & 3 & 3 \\
\hline 7377500 & 3.12 & 2.54 & 3.17 & 3.11 & 18.60 & 1.91 & $0.1 *$ & 3 & 2 & 3 \\
\hline 7378000 & 3.44 & 3.02 & 3.01 & 3.07 & 12.10 & 12.26 & $10.7 *$ & 2 & 2 & 2 \\
\hline 7378500 & 2.91 & 2.92 & 2.90 & 2.83 & 0.37 & 0.30 & 2.53 & 5 & 4 & 8 \\
\hline 7382500 & 2.67 & 2.65 & 2.81 & 2.68 & 0.82 & 5.29 & $0.75^{*}$ & 7 & 6 & 5 \\
\hline 7385500 & 0.77 & 3.17 & 3.10 & 2.99 & 75.86 & 75.21 & $74.28 *$ & 6 & 2 & 2 \\
\hline 8010000 & 3.82 & 3.04 & 3.03 & 3.03 & 20.57 & 20.59 & 20.59 & 2 & 2 & 2 \\
\hline 8012000 & 3.07 & 3.05 & 3.05 & 3.05 & 0.71 & 0.50 & $0.55^{*}$ & 4 & 4 & 8 \\
\hline 8013500 & 3.00 & 2.99 & 2.97 & 3.01 & 0.73 & 0.93 & $0.35^{*}$ & 5 & 4 & 5 \\
\hline 8014500 & 2.68 & 2.79 & 2.78 & 2.68 & 3.92 & 3.68 & $0.07 *$ & 6 & 8 & 2 \\
\hline 8015500 & 2.68 & 2.84 & 2.79 & 2.68 & 5.77 & 3.82 & $0.07 *$ & 2 & 8 & 2 \\
\hline Average & & & & & 7.79 & 7.25 & 3.07 & 4.34 & 4.29 & 3.97 \\
\hline
\end{tabular}

SRDF predicts 26 out of 38 stations value more accurate than TPCSV. The mean relative errors for the SRDF and TPCSV are $3.07 \%$ and $7.25 \%$, respectively. The number of stations which has relative errors less than $10 \%$ is 5 and 8 for the SRDF and TPCSV, respectively. After all, it is evident that PCSV and TPCSV performances are close to each other. The relative error is $7.79 \%$ for PCSV and $7.25 \%$ for the TPCSV. There is not a huge difference between two methods. However, SRDF substantially outperforms PCSV and TPCSV techniques which can be seen from Table 2 .
Although the average number of adjacent stations which are used to predict pivot station is approximately equal to each other for three methods, SRDF shows better performance than PCSV and TPCSV. Not only SRDF is a reliable method used easily in practice, but also gives more accurate predictions when compared the other techniques. 


\section{Conclusions}

In this study, 38 runoff measurement sites located in the Mississippi River Basin are used for the implementation of proposed method. The regional dependency function for each station is calculated. Regional dependency functions are obtained by TPCSV method and dependency and weighting functions are obtained by SRDF. Runoff predictions are made by using SRDF and TPCSV methods for all stations. Each station influence of radius is determined. Regional dependency function is obtained from available data. In 28 and 26 among 38 stations, the proposed approach SRDF has lower relative error than PCSV and TPCSV techniques, respectively. PCSV and TPCSV show nearly the same prediction performances. The mean relative errors for all three methods are less than $10 \%$ which are acceptable in engineering applications. The graphical and numerical criteria are employed to show the better performance of SRDF against PCSV and TPCSV. All results in the tables are interpreted and compared with each other.

Edited by: A. Shamseldin

\section{References}

Altunkaynak, A.: Significant wave height prediction by using a spatial model, Ocean. Eng., 32(8-9), 924-936, 2005.

Altunkaynak, A. and Özger, M.: Spatial significant wave height variation assessment and its estimation, J. Waterw. Port C.ASCE, 131(6), 277-282, 2005.

Altunkaynak, A., Özger, M., and Sen, Z.: Regional stream flow estimation by Standard regional dependence function approach, J. Hydraul. Eng.-ASCE, 131(11), 1001-1006, 2005.

Altunkaynak, A., Özger, M., and Şen, Z.: Triple diagram model of level fluctuations in Lake Van, Turkey, Hydrol. Earth Syst. Sci., 7, 235-244, 2003, http://www.hydrol-earth-syst-sci.net/7/235/2003/.

Huang, W. C. and Yang, F. T.: Streamflow estimation by using Kriging, Water Resour. Res., 34(6), 1599-1608, 1998.

Krige, D. G.: A statistical approach to some basic mine valuation problems on the Witwatersrand, J. Chem. Metall. Min. Soc. S. Afr., 52, 119-139, 1951.

Matheron, G.: Principles of geostastistics, Econ. Geol., 58, 12461266, 1963.

Matheron, G.: The theory of regionalized variables and Its applications, Ecole de Mines, Fontainbleau, France, 1971.

Matias, J. M., Vaamondel, A., Taboada, J., and Gonzalez-Manteiga, W.: Comparison of Kriging and neural networks with application to the exploitation of a slate mine, Math. Geol., 36(4), 463-486, 2004.

Peng, C. S. and Buras, N.: Practical estimation of inflows into multireservoir system, J. Water Res. Pl.-ASCE, 126(5), 331-334, 2000.

Öztürk, C. A. and Nasuf, E.: Geostatistical assessment of rock zones for tunneling, Tunnelling and Underground Space Technology, 17(3) , 275-285, 2002.

Şahin, A. D. and Şen, Z.: A new spatial prediction model and its application to wind records, Theor. Appl. Climatol., 79(1-2), 4554, 2004.

Şen, Z. and Habib, Z. Z.: Point cumulative semivariogram of areal precipitation in mountainous regions, J. Hydrol., 205, 81-91, 1998.

Şen, Z.: Cumulative semivariogram model of regionalized variable, Math. Geol., 21, 891-903, 1989. 